\title{
ON SYSTEMATICS AND DISTRIBUTION OF SOME SPECIES OF BRYUM (BRYACEAE, BRYOPHYTA) IN RUSSIA
}

\section{ЗАМЕТКИ К СИСТЕМАТИКЕ И ГЕОГРАФИИ НЕКОТОРЫХ ВИДОВ РОДА BRYUM HEDW. (BRYACEAE, ВRYOРНYTA) В РОССИИ}

\author{
VALERIJ I. ZOLOTOV ${ }^{1}$ \\ ВАЛЕРИЙ И. ЗОЛОТОВ ${ }^{1}$
}

Abstract

\begin{abstract}
Systematics of several species of Bryum is discussed, with the special attention to groups where the species concept was considerably expanded by the recent suggestions of Holyoak. The observations based on material from Russia support the traditional, more narrow understanding of: (1) Bryum archangelicum and Bryum amblyodon; (2) Bryum purpurascens and Bryum arcticum; (3) Bryum calophyllum and $B$. axel-blyttii. Rare species, B. teres and B. sibiricum are commented, and $B$. planiusculum is synonymized with $B$. turbinatum. New records of Bryum species for some provinces of Russia are also provided.
\end{abstract}

Резюме

Обсуждается систематика некоторых видов, которые в недавней работе Holyoak было предложено понимать значительно шире, чем традиционно. Приводятся аргументы в пользу традиционного понимания объема и дифференциальных признаков следующих пар видов: (1) Bryum archangelicum и Bryum amblyodon; (2) Bryum purpurascens и Bryum arcticum; (3) Bryum calophyllum и B. axel-blyttii. Комментируются редкие в России B. teres и B. sibiricum, а $B$. planiusculum отнесен в синонимы $B$. turbinatum. Сообщается о новых находках некоторых видов в регионах России, где они ранее не отмечались.

The genus Bryum is the largest one in the bryoflora of Russia. Many groups of it are still insufficiently known, both regarding to taxonomy and species distribution. In the course of revision of collections from the territory of Russia the new data were accumulated which are important for delimitation of several critical taxa.

Bryum archangelicum Bruch et al. and Bryum amblyodon Müll. Hal.

Bryum amblyodon (syn. B. inclinatum (Sw. ex Brid.) Turton, B. imbricatum (Schwägr.) Bruch et al.) is one of the most widespread species of Bryum in Russia, occurring in lowlands and mountain areas, on different substrates, in differently moistened habitats. It is very polymorphous, but can be recognized by the endostome fused at base with exostome, short and broad cilia (Fig. 1), large spores (18-) 24-32 (-34) $\mu \mathrm{m}$, broad leaf border, red leaf base, polyoicous sex- ual condition. In the lowland B. amblyodon is usually a large moss, having shoots up to $15 \mathrm{~mm}$ and setae up to $40 \mathrm{~mm}$. However plants from high mountains are smaller: gametophytes often don't exceed $5 \mathrm{~mm}$ tall and setae are $10-20 \mathrm{~mm}$ only. The latter is quite similar to the species recognized by Limpricht (1895), Savicz-Lyubitskaya (1954), Savicz-Lyubitskaya \& Smirnova (1970), and Nyholm (1993) as B. archangelicum. The latter differs from $B$. amblyodon in (1) aulacodontous peristome, where ventral trabeculae of exostome teeth are narrower in their middle, and these hollows of ventral trabeculae sometimes reaching the teeth body resulting in perforations or less heavily ornamented areas along median line of tooth seen as pellucid spots from the dorsal surface of tooth (Fig. 1); at the same time peristome of $B$. amblyodon is holodontous, lacking the pattern described above; (2) mature spores of $B$.

1 - Main Botanical Garden of Russian Academy of Sciences, Botanicheskaya 4, Moscow 127276 Russia - Россия 127276, Москва, Ботаническая 4, Главный ботанический сад РАН; bryum@list.ru 

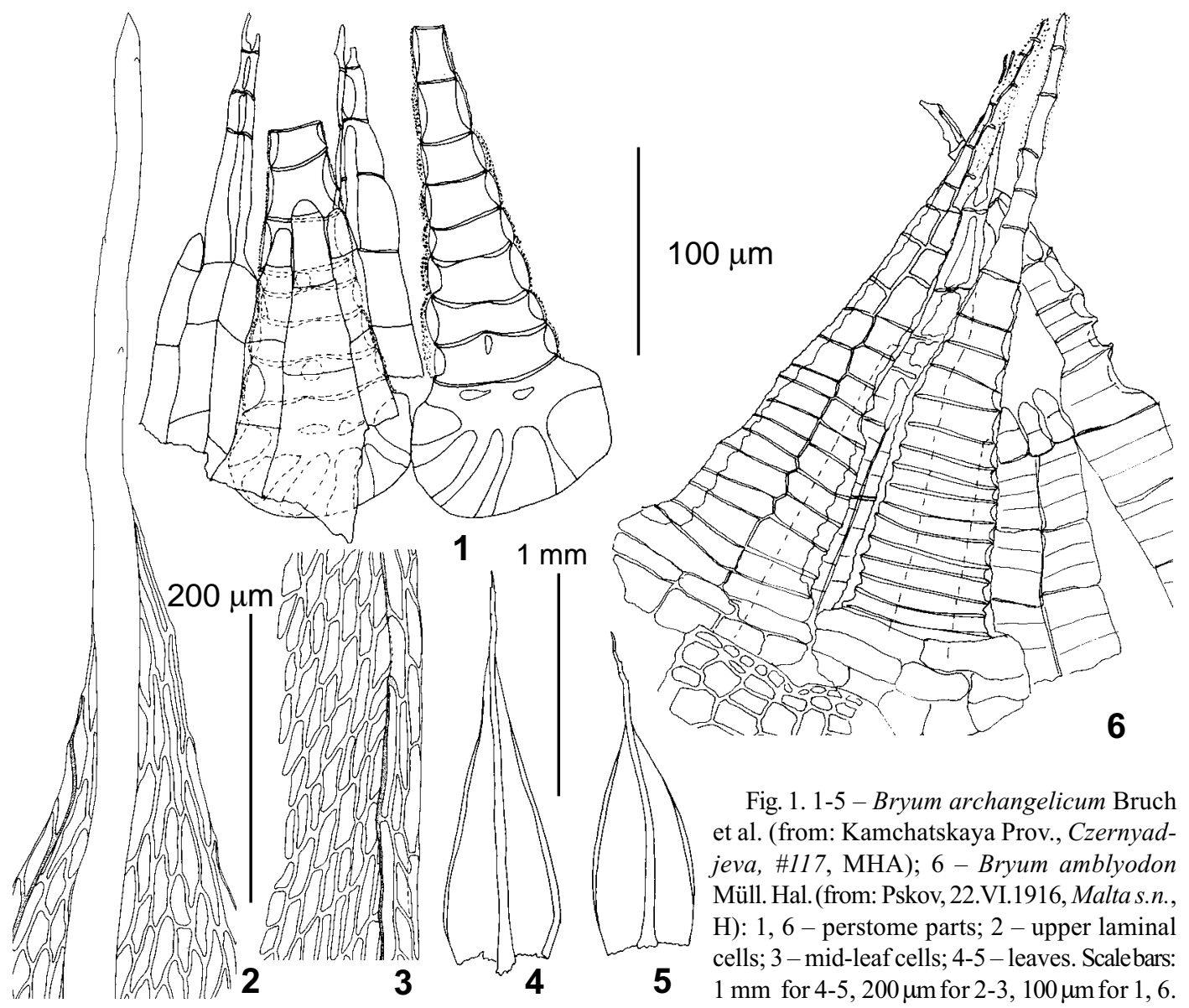

archangelicum are ferrugineous in reflexed light, orange to reddish with blackish ornamentation in compound microscope view; in B. amblyodon spores in both reflexed and transmitted light are greenish, sometimes yellowish or brownishgreen; (3) costa longly excurrent (vs. shortly excurrent in B. amblyodon); (4) leaf border inconspicuous (vs. conspicuous in $B$. amblyodon).

Holyoak (2004) synonymized $B$. amblyodon with $B$. archangelicum, considering their differences to be unstable. However in Russian material in addition to numerous specimens of $B$. amblyodon, there are plants with the stable combination of the above mentioned characters of $B$. archangelicum. The latter was observed in mountain areas of Kola Peninsula, Caucasus, Altai, Yakutia, and Kamchatka. Using this name I follow Limpricht (1895), Savicz-Lyubitskaya \& Smirnova (1970) and Nyholm (1993), as I did not study the type. Protologue of $B$. archangelicum mentions "Sporae ma- jusculae, ferrugineae" but provide no information about aulacodontous peristome (which I consider as a main diagnostic character); however this character was not used at all by Bruch et al. (1846). Limpricht (1895) clearly illustrated aulacodontous peristome of $B$. archangelicum, but it has to be still prooved that this agree with the type of this species. Holyoak (2004) did not mention if he studied the types of $B$. archangelicum and B. amblyodon. So, at moment, I continue to keep these two species separately.

Specimens examined:

Bryum archangelicum: Altai Republic: Altai Mts., Kobiguayuk Creek, $2220 \mathrm{~m}$ alt., Ignatov \#0/108(MHA); Kamchatskaya Province: Czernyadjeva \# 53, \#117, 22.VIII.2004 (LE); 19.VIII.2001, Czernyadjeva \#116 (LE); Czernyadjeva \#19, 16.VII.2003 (LE); 22.VII. 2003, Czernyadjeva \#54 (LE); Karachaevo-Cherkessian Republic: Teberda Nature Reserve, $1300 \mathrm{~m}$ alt., Zolotov, 5.IX.2005 (MHA); $3150 \mathrm{~m}$ alt., Ignatov \& Ignatova \# 05-3639 (MHA, MW); Murmansk Province, 
Terskij shore of White Sea, Andreeva, 15.VII.1984 (KPABG); Kandalaksha Reserve, Belkina \& Likhachev \# 319/2 (KPABG); Taimyrsky Autonomous District: Medusa Bay, Varlygina, 11.VII.2003 (MHA, MW); Republic Sakha/Yakutia: Suntar-Khayata Reserve, $760 \mathrm{~m}$ alt., Zolotov \& Ivanova, 18.VII.2003 (MHA).

Bryum amblyodon: Altai Republic: Krylov, 23.VI.1903 (MHA, LE); Ignatov \& Ignatova \# 25/61 (MHA); Ignatov, 4.VIII.1992 (MHA); Republic Bashkortostan: Selivanova-Gorodkova \# 2 (LE); Podpera, 10.VIII.1917 (LE); Republic Buryatia: Tubanova \# 5(VII), 37(V), 33(V) (UUH); Sakhalinskaya Province: Iturup Island, Bakalin \# K-44-17-05 (MHA, VLA); Shumshu Island, Bakalin \# K-128-04 (MHA, VLA); Republic Kabardino-Balkaria: Ignatov et al., 31.VII. 2004 (MHA); Kamchatskaya Province: 19.VIII.2005, Czernyadjeva \#41 (LE); 19.VIII. 2001, Czernyadjeva \#116 (LE); Czernyadjeva \#47, 6.VIII. 2002 (LE); Czernyadjeva \#53, 21.VII.2003 (LE); Czernyadjeva, \#65 (LE); Czernyadjeva, 2.VIII.2005 (LE); KarachaevoCherkessian Republic: Teberda Nature Reserve, Dombai, 2000 m alt., Zolotov, 10.IX.2005 (MHA); same place, $3000 \mathrm{~m}$ alt., Zolotov, 10.IX.2005 (MHA); same place, $3100 \mathrm{~m}$ alt., Zolotov, 10.IX.2005 (MHA); Teberda Nature Reserve Dzhamagat River valley, Zolotov, 12.IX.2005 (3 specimens) (MHA); Teberda Nature reserve, Malaja Khatipara Mt. Range, Zolotov, 2.IX.2005 (MHA); same place, Zolotov, 3.IX.2005 (2 specimens) (MHA); Teberda Nature Reserve, Ullu-Murudzhu River valley, Zolotov, 14.IX.2005 (MHA); Moscow Province: Zickendrath \# $221(\mathrm{H})$; Murmansk Province: Belkina \# 35-17-99 (8381), 587-8-04, 1-6-99, 28-5-86, 51-13-01 (10490), 51-12-01 (10489), 51-14-01 (10491), 51-10-01 (10487), 82-01 (10594) (KPABG); Schljakov \# 1804, 1806, 1894, 1921 (KPABG, LE); Schljakov \# 88, 145, 237, 252a, 275, 349, 429, 588, 611 (RS611-72), RS-4-50 (KPABG); Likhachev \# 40-6-91, 218 $5-91,212-1-91,102-8-91,54-1-91,220-8-91,102-1-$ 91, 355-6-88, L12-4-97 (5795), 202-25-89 (3310) (KPABG); Dombrovskaja \# 163-67 (8565) (KPABG); Belkina \& Likhachev \# 373/3, 417-9-88 (6756), 218-389 (3338), 323/12, 391-6-88 (KPABG); Pskov Province: Malta, 22.VI.1916 (LE); Sakhalinskaya Province: Sakhalin Island, Barkalov, 3.VIII.2001 (MHA, VLA); Tomsk Province: Dokturovskij (MHA); Vologda Province, Kirillov town, 22.VII.1967, 31.VII. 1967 (MHA); Taimyrskij Autonomous District: Western Taimyr, Varlygina, \# 57 (MW, MHA); same place, Varlygina, 25.VII.2002 (MW, MHA); Republic Sakha/Yakutia: Kildjushewskij, \#3/5 (LE); Zolotov \& Ivanova, \# 00-01, 02-01, 02-02, 02-03, 16-01, 25-03, 28-01, 41-01, 41-02, 46-09 46-12, 46-16 (MHA); $\mathrm{Ni-}$ kolin, 28.VI. 1987 \& 13.VI. 1990 (SASY); Barychev, 7.VII.1990 (SASY).
Bryum purpurascens (R. Br.) Bruch et al. and Bryum arcticum (R. Br.) Bruch et al.

Holyoak (2004) synonymized these two species because he found that the cross-striolate ornamntation of outer surface of exosiome in B. purpurascens is intergrading to reticulate pattern which is characteristic for $B$. arcticum (and most other Bryum species). These two character states, however, are always quite distinct in Russian collections. Beside the ornamentation of the outer surface of exostome, there is one more stable difference in the structure of the inner surface of teeth: in Bryum arcticum there are septae between the ventral trabeculae, that are absent in B. purpurascens.

In addition, cilia of $B$. arcticum are always rudimental, while in B. purpurascens they are often quite well-developed. This character, of course, can not be used alone because in species with usually reduced cilia, i.e. B. amblyodon and B. algovicum, more developed and even appeniculate cilia are sometimes observed (althought this can be observed usually on just a single cilium). Summing up, Russian material provides no problem for to be sorted into B. purpurascens and B. arcticum, and thus we suggest to keep these species separately.

Specimens examined:

B. purpurascens: Murmansk Province: Poljanskaja \& Gutovskij \# 327, 13.VI. 1939 (LE); Sorokin, 08.VIII. 2002 (MHA); Schljakov, \# 281, 1039 (KPABG); Likhachev, \# 34/5, 247/12 (KPABG); Brotherus, 14.VIII.1885 (LE); Kola, Brotherus, VII.1885(LE); Vaidoguba, Brotherus, VIII.1885 (LE); Lubovi, Brotherus, VII.1885 (LE); Kuusamo, Brotherus, 17.VII.1883 (LE); Kihlman \# 215, 14.VII.1889 (LE); Komi Republic: Kildjushevskij, Zheleznova \& Frolova, 2. VII.1970 (SYKO); Yamalo-Nenetskiy Autonomous District: Salekhard, Soldatenkova, 21.IX.1964 (MHA).

Bryum arcticum: Murmansk Province: Schljakov \# 1195 (KPABG, LE); Likhachev \# 4-1-78 (9263) (KPABG); Belkina \#4-1-97 (5727), O53-1-85 (11877) (KPABG); Karachaevo-Cherkessian Republic: Dombai, Mussa-Achitara Mt., $3000 \mathrm{~m}$ alt., Zolotov, 10.IX.2005 (MHA); Taimyrskij Autonomous District: Pospelova \# 94/30, VIII.1994 (MW, MHA); North-Eastern Taimyr, $75^{\circ} 15^{\prime} \mathrm{N}-112^{\circ} 30^{\prime}$ E, Pospelova \# 57, 16.VII.1992 (MW MHA); Western Taimyr, Varlygina, 3.VII.2003 (MW, MHA); Varlygina, 4.VII.2002 (MW, MHA) (3 specimens); Varlygina,5.VII.2003 (MW, MHA); Varlygina, 25.VII.2003 (MW, MHA); Varlygina, 25.VII.2003 (MW, MHA); Varlygina, 6.VII.2003 (MW, MHA); Varlygina, 


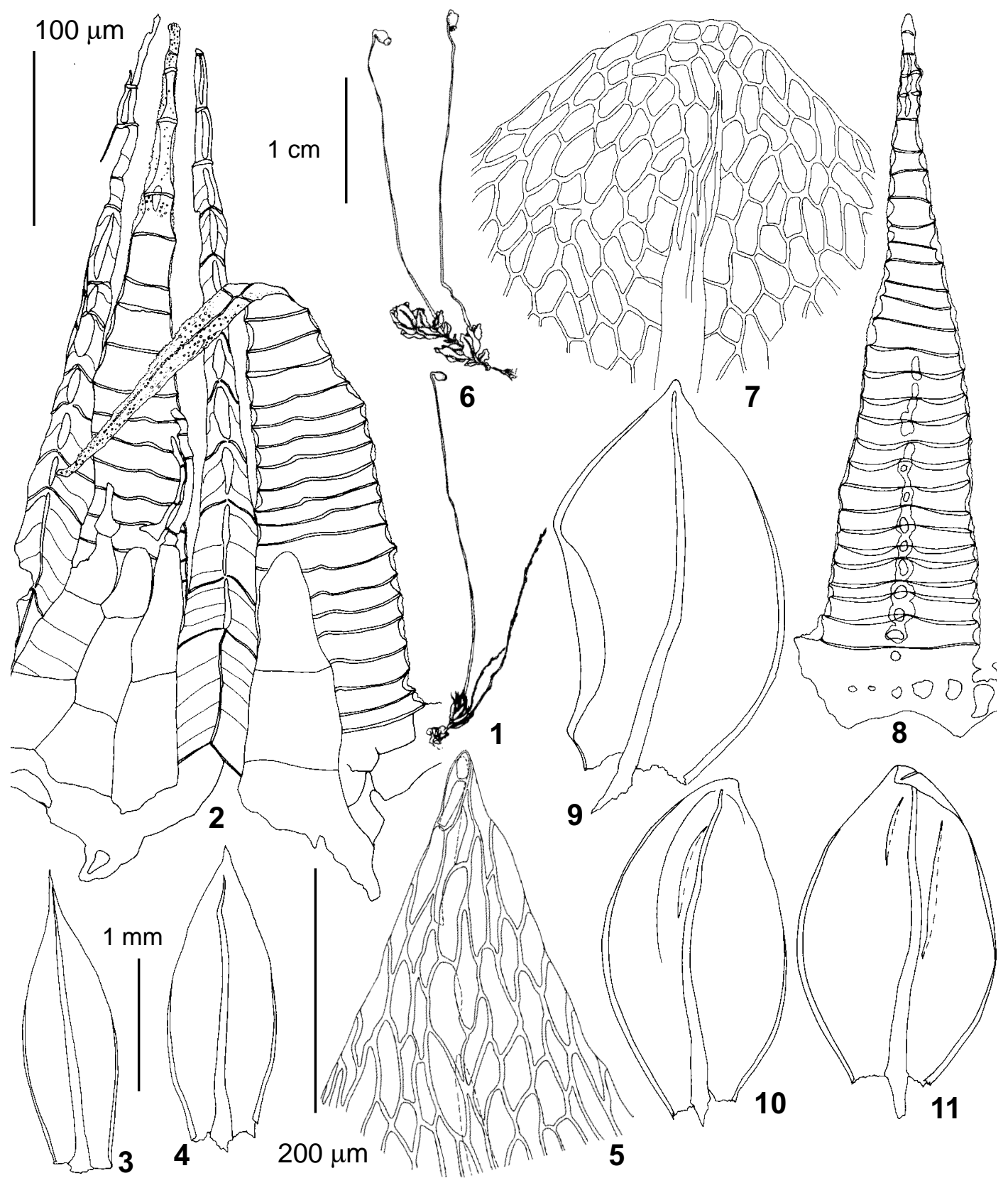

Fig. 2. 1-5 - Bryum axel-blyttii Kaurin ex H. Philib. (from Krasnoyarsk Territory, 20.VIII.1992, Otnyukova s.n., MHA); 6-11 - B. calophyllum R. Br. (from Taimyr Peninsula, Pospelova, \# 494/152, MHA): 1, 6 - habit; 2, 8 peristome parts; 5, 7 - upper laminal cells; 3-4, 9-11 - leaves. Scale bars: $1 \mathrm{~cm}$ - for 1,6; $1 \mathrm{~mm}$ for 3-4, 9-11;200 $\mu \mathrm{m}$ for $5,7,100 \mu \mathrm{m}$ for 2,8 .

VII.2002 (MW, MHA); Varlygina, 8.VII.2002 (MW, MHA); Republic Buryatia: Tubanova, \# 20(VII) (UUH); Republic Sakha/Yakutia: 14.VII.2003, Zolotov \& Ivanova, \# 36-05 (MHA); 19.VII.2003, Zolotov \& Ivanova, \# 42-01 (MHA); Kobjajskij Distr., Ivanova, 12.VI. 1990 (SASY) (2 specimes); Bulunskij
Distr., Perfiljeva, 14.VII.1987 (SASY); Laptevykh Sea coast, Stepanova, 7.VIII.1981 (SASY).

Bryum calophyllum $\mathrm{R}$. Br. and B. axel-blyttii Kaurin ex H. Philib.

Bryum axel-blyttii was synonymized with $B$. 

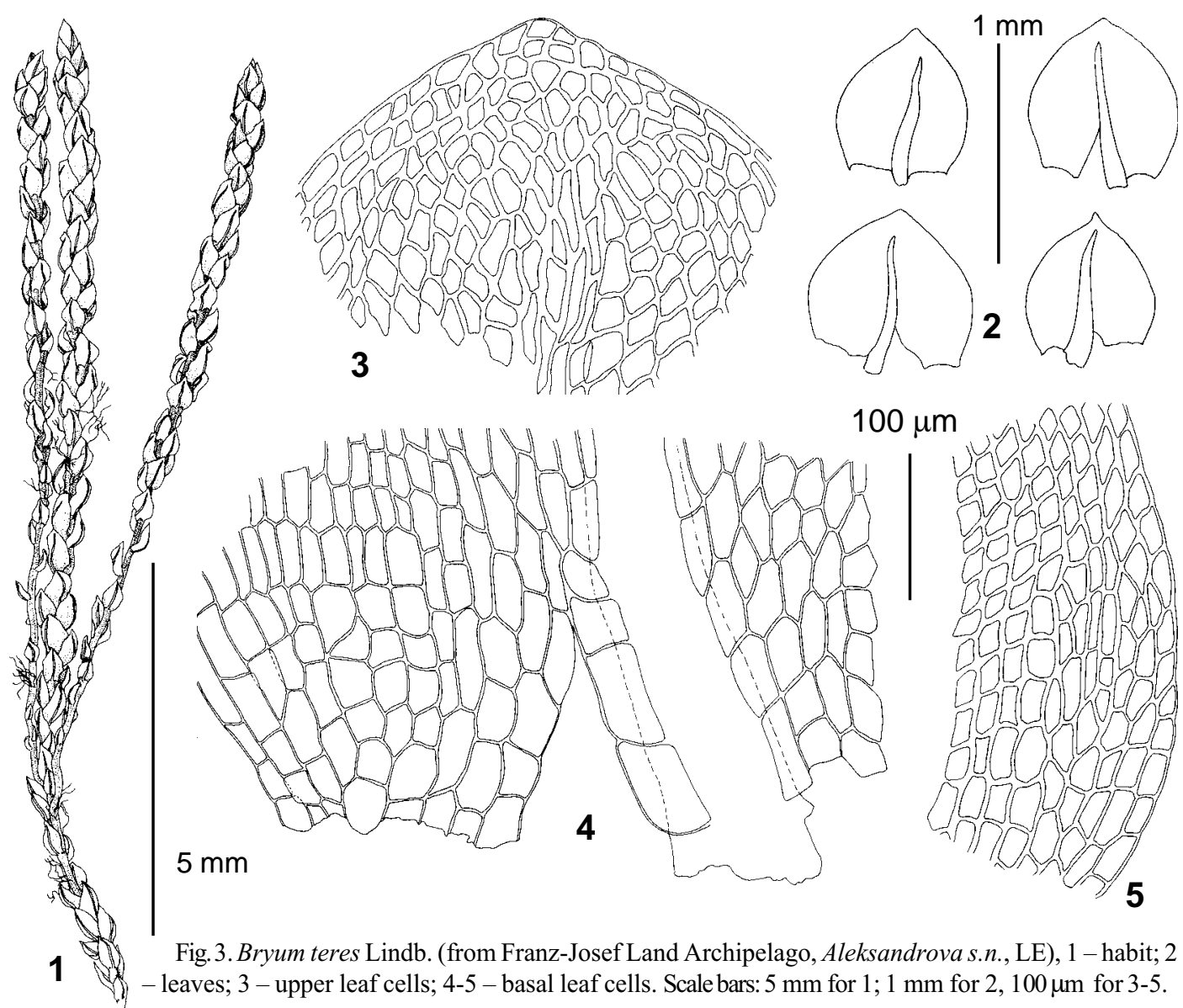

\section{3}

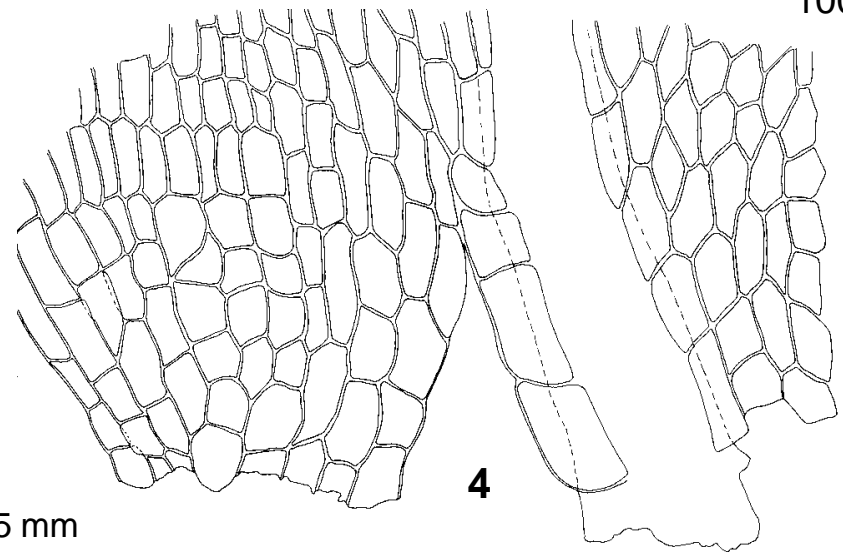

$100 \mu \mathrm{m}$

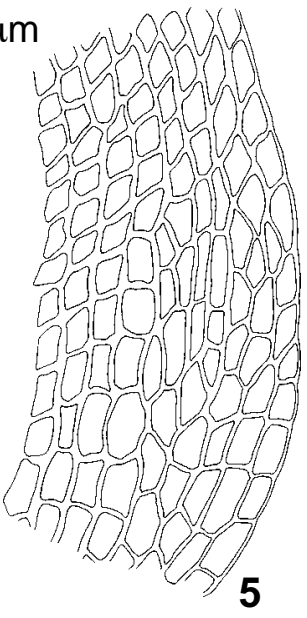

Fig. 3. Bryum teres Lindb. (from Franz-Josef Land Archipelago, Aleksandrova s.n., LE), 1 - habit; 2 - leaves; 3 - upper leaf cells; 4-5 - basal leaf cells. Scale bars: $5 \mathrm{~mm}$ for $1 ; 1 \mathrm{~mm}$ for 2, $100 \mu \mathrm{m}$ for 3-5.

calophyllum by Holyoak (2004). According to relatively few specimens seen from Russia, however, they lack intermediate plants and can be separated. Both species are rather rare in Russia, especially $B$. axel-blyttii. The latter differs from the more common B. calophyllum in narrower leaves with acute (vs. obtuse) leaf apex, and no or few perforation along the median line of teeth (vs. numerous perforations in B. calophyllum) (Fig. 2). Thus, as in the case of $B$. archangelicum- $B$. ablyodon, the studied material provides no evidences for necessity of combining these species.

Specimens examined:

B. axel-blyttii: Krasnoyarsk Territory, Lama Lake near mouth of Mikchanda Creek, $80 \mathrm{~km}$ NE from Norilsk $\left[68^{\circ}-70^{\circ} \mathrm{N}, 85^{\circ}-90^{\circ} \mathrm{E}\right]$, Larix forest with Betula nana, within moss carpet, Otnyukova, 20.VIII.1992 MHA; Republic Sakha/Yakutia, Ust-Maya District, Allakh-Yun, $61^{\circ} 08^{\prime} \mathrm{N}-138^{\circ} 03^{\prime} \mathrm{E}$, willow thicket along river bar, Ignatov, 23.VIII.2000 [MHA];
B. calophyllum:Taimyrsky Autonomous District: Taimyr Peninsula, Lake Syrutaturku, $73^{\circ} 35^{\prime} \mathrm{N}-$ $97^{\circ} 30^{\prime} \mathrm{E}$, successional grassland on somewhat salty landslide, Pospelova, \# 494/152 (MHA); Severnaya Zemlya Archipelago: Oktyabrskoy Revolyutsii Island, Zhiloy Peninsula, $78^{\circ}-80^{\circ} \mathrm{N}, 93^{\circ}-100^{\circ} \mathrm{E}$, spotty tundra, Khodachek, 24.VII.1975 (LE).

\section{Bryum teres Lindb.}

This species was reported in many regional lists of mosses, but my study of collections in KPABG, LE, SASY revealed that a lot of so-named specimens belong to other species which formed thin shoots due to unfavorite conditions. Carefull study of lower leaves (sometimes already buried in peat) in such collections often allows to find bigger leaves, sometimes allowing to identify species, e.g. B. elegans, B. neodamense, B. pseudotriquetrum, and even $B$. bicolor, $B$. intermedium, B. amblyodon, etc. At the same time, B. teres has a number of stable characters: plants in loose tufts, 
easily separating, without rhizoidal tomentum; shoots gracilous, leaves closely imbricate, although they are only slightly overlapping when dry; shoots about $1 \mathrm{~cm}$ long, less than $1 \mathrm{~mm}$ wide; stem dark-red to black, rigid, fragile (when both dry and wet - so the attempt of leaf detachment often results in fragmentation of stem with leaves); upon drying stem remains straight (neither curved, nor twisted); leaves broadly ovate, with length to width ratio about $1: 1$, concave, obtuse, non-decurrent, red at base (and sometimes up to halfleaf), $1 \mathrm{~mm}$ long, leaf margin plane, not bordered; costa vanished below leaf apex; upper leaf cells thick-walled (Fig. 3). These characters can be found in some young sterile plants of other species, but the obviously adult plants that can be certainly attributed to $B$. teres are very rare: only two collections were found in herbaria.

Specimens examined: Arkhangelsk Province: Franz-Josef Land Archipelago, Island Zemlya Aleksandry, $80^{\circ}-85^{\circ} \mathrm{N}, 45^{\circ}-65^{\circ} \mathrm{E}$, along a brook, Aleksandro$v a$ (LE); Chukotskiy Autonomous District: Vrangel Island, Rodgers Bay, $71^{\circ}-72^{\circ} \mathrm{N}, 178^{\circ}-183^{\circ} \mathrm{E}$, mouth of Nasha River, solty place at sea shore, Afonina, 21.IX.1987 (LE).

Bryum turbinatum (Hedw.) Turn., Muscol. Hibern. Spic. 127. 1804. — Bryum planiusculum Lindb.et Arnell., Kongl. Svensk. Vetenskapsakad. Handl. 23(10): 38. 1890. syn. nov. Holotype: “Type! Post mortem Lindbergii insertus. + , Bryum planiusculum n. sp., Sibiria, Potkamina Tunguska, $61^{\circ}$ 30' Sept. 28/76, Arnell"' (H-SOL 0489001!). Isotype (H-BR 0681011!).

Bryum planiusculum was described by a female plants without sporophyte. The type specimens has small yellowish-green tufts of female plants $0.4-1.0 \mathrm{~cm}$ tall, evenly foliated, rather opaque; leaves lanceolate, appressed, rigid, keeled, almost non-decurrent, without red color in proximal leaf; costa strong, excurrent; cells in mid-leaf 42-70x15-20 $\mu \mathrm{m}$; leaf border uniseriate, entire or slightly serrulate above.

No one of plant characters contradict to female plants of B. turbinatum.

\section{Bryum sibiricum Lindb. \& Arnell}

According to Lindberg \& Arnell (1890) this species differs from the closely related B. pallens in non-decurrent, more narrow and more longly acuminate leaves, 4-5-seriate leaf border, longer and narrower urn, and larger exothecial cells. The only specimen which perfectly agree with the original description was found. It is rather contrastingly different from $B$. pallens which is relatively little variable in gametophytic characters. It is possible that $B$. sibiricum is a good, but very rare species. Further studies of this species are needed.

Specimen examined: Republic Sakha/Yakutia, Khangalasskiy Dictrict, between Kytyl Dzhura and Krestyakh, NW-faced slope, Larix forest, coll. Zakharova, 14.VII.1989, det. Kazanovsky, conf. Zolotov (SASY).

NEW RECORDS OF BRYUM IN RUSSIA

During work on the updated check-list of mosses of the East Europe and North Asia, the lack of data for certain species from some areas was revealed. Thus, the new records for certain areas are listed below:

Bryum bicolor Dicks. - Severnaya OssetiyaAlania Republic: Mozdok District, Kizlyar, 43 $43^{\prime} \mathrm{N}$ $-44^{\circ} 35^{\prime} \mathrm{E}, 70-170 \mathrm{~m}$ alt., abolished fields and roadsides, Zolotov, 5.V.2006-10.V.2006 (MHA); Stavropol Territory: Georgievsk, $44^{\circ} 07^{\prime} \mathrm{N}-43^{\circ} 26^{\prime} \mathrm{E}, 260-$ $300 \mathrm{~m}$ alt., abolished fields and roadsides, Zolotov, 5.V.2006-10.V.2006 (MHA).

B. bimum (Hedw.) Turn. - Sakhalinskaya Province: Iturup Island, Chernye Skaly $45^{\circ} 15^{\prime} 32,1^{\prime \prime} \mathrm{N}$ $-148^{\circ} 10^{\prime} 22,9^{\prime \prime} \mathrm{E}, 15 \mathrm{~m}$ alt., crevices of rocks under Alnus canopy, Bakalin, \# K-66-11-05 (MHA, VLA).

B. capillare Hedw. - Sakhalinskaya Province: Iturup Island, $45^{\circ} 15^{\prime} 01,8^{\prime \prime} \mathrm{N}-148^{\circ} 01^{\prime} 11,7^{\prime \prime} \mathrm{E}, 20 \mathrm{~m}$ alt., on soil in forest on steep slope, Bakalin, \#K65-1-05 (MHA, VLA).

B. cryophilum Mårtensson - Taimyrskiy Autonomous District: Taimyr Reserve, Central Byrranga Range, B. Bootankagi River in middle course, near Vetvistyi Creek mouth, $76^{\circ} 22^{\prime} \mathrm{N}-98^{\circ} 05^{\prime} \mathrm{E}$, dry brook bed, on alluvium, Pospelova, \# 170 (MHA).

B. elegans Nees ex Brid. - Taimyrskiy Autonomous District: West Taimyr, the Big Arctical State Reserve, Meduza Bay, $73^{\circ} 22^{\prime} \mathrm{N}-80^{\circ} 32^{\prime} \mathrm{E}$, rocky ridge, on rocks and among them, Varlygi$n a$, 5.VII.2003 (MHA, MW).

B. klinggraeffii Schimp. - Moscow Province: Moscow, nature park "Valley of Skhodnya River in Kurkino", 55 $52,665^{\prime} \mathrm{N}-37^{\circ} 23,225^{\prime} \mathrm{E}$, edge of Betula forest, on soil above concrete block, Zolotov, 9.XII.2006 (MHA); same place, [55 $54,634^{\prime} \mathrm{N}$ $\left.-37^{\circ} 23,080^{\prime} \mathrm{E}\right]$, high bank of stream Mashkinsky, 


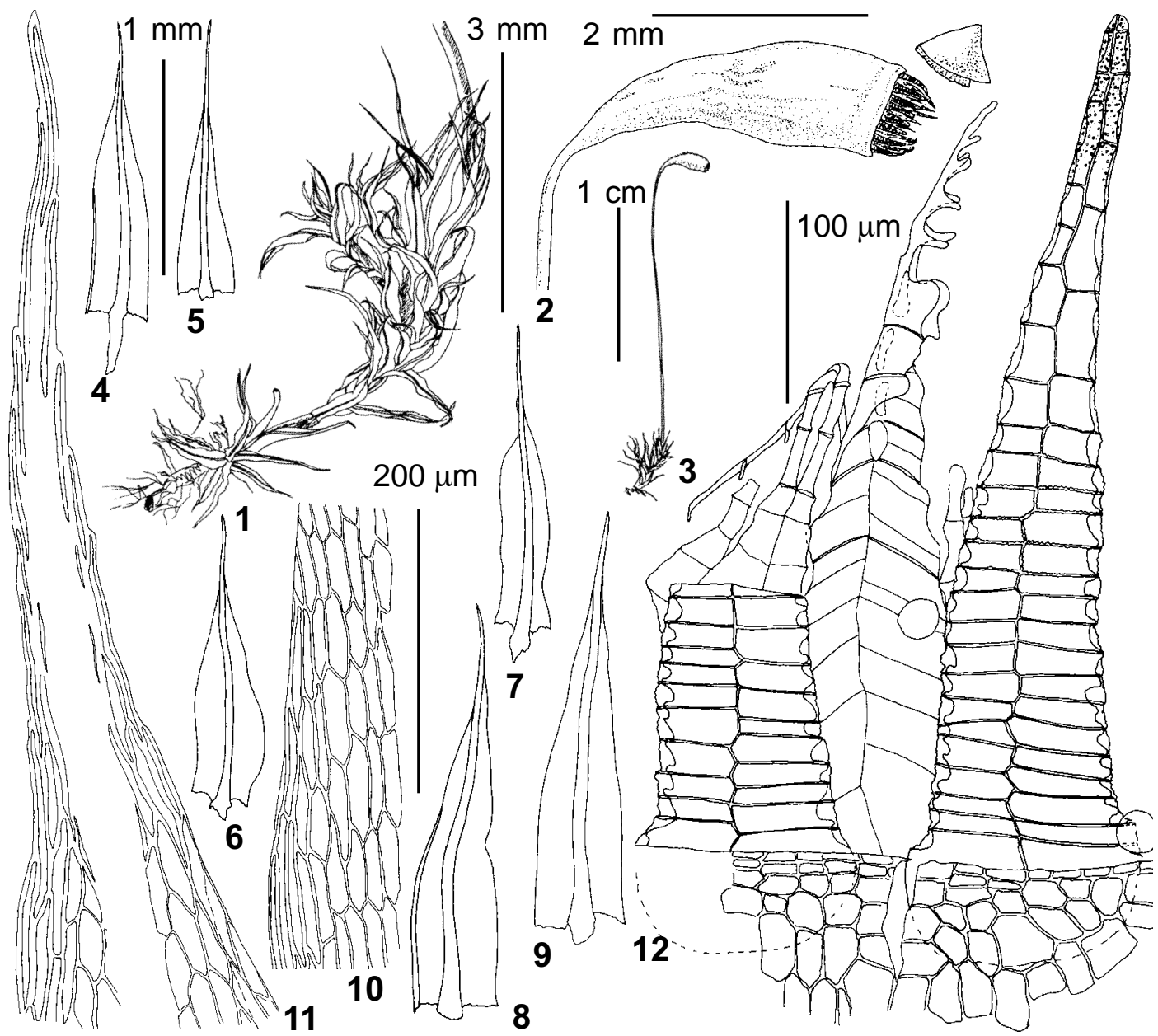

Fig. 4. Bryum sibiricum Lindb. \& Arnell (from: Yakutia, 14.VII.1989, Zakharova s.n., SASY): 1, 3 - habit; 2 capsule; 4-9 - leaves; 10 - mid-leaf cells; 11 - upper laminal cells; 12 - part of peristome. Scale bars: $1 \mathrm{~cm}$ - for 3;3 $\mathrm{mm}$ for $1 ; 2 \mathrm{~mm}$ for $2 ; 1 \mathrm{~mm}$ for $4-9 ; 200 \mu \mathrm{m}$ for $10-11 ; 100 \mu \mathrm{m}$ for 12 .

on soil among grass, Zolotov, 11.XII.2006(MHA).

B. knowltonii Barnes - Chukotskiy Autonomous District: Pekulnei range, upper reach of Bychja River, $65^{\circ} 57^{\prime} \mathrm{N}-174^{\circ} 37^{\prime} \mathrm{E}$, on rocks along stream, Korobkov, 27.VIII.1977 (LE); Taimyrskiy Autonomous District: Lake Syrutaturku, $73^{\circ} 35^{\prime} \mathrm{N}$ - 97 $30^{\prime} \mathrm{E}$, grass + Dryas tundra near stream, Pospelova, VII.1994 (MHA, MW); West Taimyr, the Big Arctic State Reserve,Meduza Bay, $73^{\circ} 22^{\prime}$ $\mathrm{N}-80^{\circ} 32^{\prime} \mathrm{E}$, on eroded soil, Varlygina, 11.VII.2003 (MHA, MW).

B. lonchocaulon Müll. Hal. - Chukotskiy Autonomous District: Pekulnei range, upper reach of Bychja River, $65^{\circ} 57^{\prime} \mathrm{N}-174^{\circ} 37^{\prime} \mathrm{E}$, пологий шлейф, бугорковатая кустарничково-осоковая тундра, Korobkov, 26.VII.1971 (LE).

B. longisetum Blandow ex Schwägr. - Altai Republic: Altai Mts., Saluru Creek, 50³4' N $89^{\circ} 06^{\prime} \mathrm{E}$, on spots of mineral soil, Ignatov, 1.VIII.1993 (MHA).

B. marratii Wilson - Altai Republic: Altai Mts., Tokpak Creek ( $8 \mathrm{~km}$ north of Kosh-Agach), 5004' N $-88^{\circ} 39^{\prime} \mathrm{E}, 1980 \mathrm{~m}$ alt., springy bog aroung a lake in Chuiskaya steppe, Ignatov, 21.VII.1993 (MHA).

B. mildeanum Jur. - Altai Republic: Altai Mts., Shebalino District, Elekmonar Creek Valley, $25 \mathrm{~km}$ upstream from the Katun River, 50 $34^{\prime} \mathrm{N}-89^{\circ} 06^{\prime}$ $\mathrm{E}, 1100 \mathrm{~m}$ alt., along road across pasture Ignatov \& Ignatova, 4.VIII.1991, (MHA). 
B. neodamense Itzigs. - Kamchatskaya Province: Elizovo Distr., $14 \mathrm{~km} \mathrm{SW}$ of agricultural station, Bystraya River, on rocks near water, 5.XI.1961, Leshchina s.n. (MHA); Taimyrskiy Autonomous District: Western Taimyr, Meduza Bay, $73^{\circ} 22^{\prime} \mathrm{N}-$ $80^{\circ} 32^{\prime} \mathrm{E}$, Meduza River, wet place in valley, Varlygina, 18.VII.2002 (MHA, MW).

B. oblongum Lindb. - Vologda Province: Sukhona River near Nyuksenizy, $60^{\circ} 24^{\prime} \mathrm{N}-44^{\circ} 11^{\prime} \mathrm{E}$, right bank, line cutting of coniferous forest, Ignatov \& Ignatova, 13.VIII.2001 (MHA).

B. radiculosum Brid. - Sakhalinskaya Province: Iturup Island, $49^{\circ} 09^{\prime} 00,2^{\prime \prime} \mathrm{N}-147^{\circ} 57^{\prime} 49,8^{\prime \prime} \mathrm{E}$, 402 m alt., Juncus-Carex-shrublets-mossy mire in the uppermost course of Kurilskaya River, hollows between Carex hummocks, Bakalin, \# K49-11-05 (VLA, MHA).

B. rubens Mitt. - Leningrad Province: Boxitogorsk Distr., 59³2'20,7'N - 3401'50,6"E, along road in forest, on soil, Zolotov, 9.VIII.2004 (MHA); Stavropol Territory: Georgievsk, $44^{\circ} 05^{\prime} 52,8^{\prime \prime} \mathrm{N}-$ $43^{\circ} 26^{\prime} 27,7^{\prime \prime E}, 276.3 \mathrm{~m}$ alt., waste field, on soil, Zolotov, 11.V.2006 (MHA); Severnaya OssetiyaAlania Republic: Mozdok Town surroundings, $43^{\circ} 44^{\prime} 24,6^{\prime \prime} \mathrm{N}-44^{\circ} 35^{\prime} 27,9^{\prime \prime} \mathrm{E}, 161.7$ malt., waste field, on soil, Zolotov, 7.V.2006 (MHA).

B. salinum I.Hagen ex Limpr. - Taimyrskiy Autonomous District: Western Taimyr, the Big
Arctic State Reserve, Medusa Bay, $73^{\circ} 22^{\prime} \mathrm{N}-80^{\circ} 32^{\prime}$ E, wet bank of stream, Varlygina, 15.VII.2001 (MHA, MW).

B. savicziae Schljakov - Altai Republic: Altai Mts., Tokpak Creek in middle course, $50^{\circ} 12^{\prime} \mathrm{N}-$ $88^{\circ} 52^{\prime} \mathrm{E}, 2050 \mathrm{~m}$ alt., on boulder in the creek, above water, Ignatov, 23.VII.1993 (MHA).

B. subapiculatum Hampe - Leningrad Province: Boxitogorsk District, 59०32'20,7"N $34^{\circ} 01^{\prime} 50,6^{\prime \prime} \mathrm{E}$, на обочине лесной дороги, на почве, Zolotov, 9.VIII.2004 (MHA).

B. turbinatum (Hedw.) Turner - Republic Buryatia: Kurumkanskiy Distr., Dzerginskiy Reserve, Tubanova \#9(X), 28.IV.1999 (UUH).

B. violaceum Crundw. \& Nyholm - KhantyMansijskij Autonomous District: Kogalym town, ruderal vegetation on west edge, on bare loam, Bezgodov \# 26 (MHA); same place, humificated sand, Bezgodov \# 111 (MHA).

\section{ACKNOWLEDGEMENTS}

I am grateful to O.M.Afonina, I.V. Czernyadjeva, V.A.Bakalin, A.G.Bezgodov, D. Leshchina, E.A.Ignatova, D.Ya.Tubanova, T.I.Varlygina for making to my disposal their collections, to curator of $\mathrm{H}$ for loan of type material of Bryum planiusculum. I am grateful to M.S.Ignatov for help in paper preparing. The work was supported by RFBR 04-04-48774.

\section{LiTERATURE CITED}

BRUCH, PH., W.Ph. SCHIMPER \& Th.GÜMBEL 1846. Bryologia Europaea seu genera muscorum Europaeorum monographice illustrata. 4. - Stutgartiae, Sumptibus Librariae E. Schweizerbart.

HOLYOAK, D.T. 2004. Taxonomic notes on some European species of Bryum (Bryopsida: Bryaceae). $-J$. Bryol. 26: 247-264.

LIMPRICHT, K.G. 1895. Die Laubmoose. - In: Rabenhorst, L. Kryptogamen-flora von Duetschland, Oesterreich und der Scheiz. Bd. 3. 1-448.

LINDBERG, S.O. \& H.W. ARNELL 1890. Contributio ad floran cryptogamam Asiae boreali-orientalis. - Acta Soc. Sci. Fennicae 10: 221-280.

NYHOLM, E. 1993. Illustrated flora of Nordic mosses, Fasc. 4. Aulacomniaceae - Meesiaceae - Catoscopi- aceae - Bartramiaceae - Timmiaceae - Encalyptaceae - Grimmiaceae - Ptychomitraceae - Hedwigiaceae Orthotrichaceae. Copenhagen \& Lund: Nordic Bryological Society.

[SAVICZ-LYUBITSKAYA, L.I.] САВИЧ-ЛЮБИЦКАЯ Л.И. 1954. Обзор рода Bryum Hedw. в СССР. - [An overview of genus Bryum Hedw. in the USSR] Tp. бот. ин-та АН СССР, сер. 2. Споровые растения [Trudy Bot. Inst. Akad. Nauk SSSR, ser. 2. Sporovye Rasteniya] 9: 495-634.

[SAVICZ-LYUBITSKAYA, L.I. \& Z.N. SMIRNOVA] САВИЧ-ЛЮБИЦКАЯ Л.И., З.Н. СМИРНОВА 1970. Определитель листостебельных мхов СССР. Верхоплодные мхи. - [Handbook of mosses of the USSR. The acrocarpous mosses] Л., Наука [Leningrad, Nauka], 822 . 